\title{
ON THE SOLUTION OF 3-D FREQUENCY DEPENDENT CRANK-NICOLSON FDTD SCHEME
}

\author{
H. K. Rouf and F. Costen
}

School of Electrical and Electronic Engineering The University of Manchester

Sackville Street, Manchester M60 1QD, UK

\section{S. G. Garcia}

Department of Electromagnetism and Matter Physics University of Granada Granada 18071, Spain

\section{S. Fujino}

Research Institute for Information Technology

Kyushu University

6-10-1 Hakozaki, Higashi-ku, Fukuoka 812-8581, Japan

\begin{abstract}
Unconditional stability of the Crank-Nicolson Finite Difference Time Domain (CN-FDTD) method permits us to use time steps over the Courant-Friedrich-Lewy (CFL) limit of conventional FDTD method. However, in this work it was realized that, when the time step is set above CFL limit the coefficient matrix arising from Crank-Nicolson method is no longer diagonally dominant and iterative solvers require longer solution time in each FDTD iteration. Frequency dependent CN-FDTD (FD-CN-FDTD) scheme for Debye media is formulated and numerical tests are performed with two widely used sparse iterative solvers, Bi-Conjugate Gradient Stabilised (BiCGStab) and Generalised Minimal Residual (GMRES), for comparison. BiCGStab outperforms GMRES in every aspect of the study.
\end{abstract}




\section{INTRODUCTION}

The Finite Difference Time Domain (FDTD) [1] method is one of the most widely used numerical techniques used in computational electrodynamics. FDTD is straightforward, robust, versatile and widely applicable for broadband systems. In such systems applications can involve numerical modeling of interaction with frequencydispersive materials [2] with the modification of the original FDTD scheme [3-6]. However, for many problems of interest it may become computationally inefficient, due to the upper limit for the time step imposed by the Courant-Friedrich-Lewy (CFL) stability condition [7]. This typically happens when a high spatial resolution is required to model fine geometries. This leads to spatial sampling much shorter than the minimum wavelength of interest, enforcing an unnecessarily large FDTD iteration numbers $[8,9]$. There is a growing interest in overcoming this limitation by employing unconditionally stable implicit FDTD methods, for which time and space steps can be independently chosen $[10,11]$. This trend will continue because high accuracy in modeling is increasingly in demand with the massive improvement of computational resources. An alternative to the explicit FDTD is the Crank-Nicolson [13] FDTD (CN-FDTD) method which is unconditionally stable beyond the CFL limit. As in the classical FDTD, CN-FDTD replaces the time and space derivatives by second order centred differences, but unlike FDTD, the fields affected by the curl operators are also averaged in time. The resulting scheme is an unconditionally stable fully implicit marching-on-in-time algorithm with high accuracy and low anisotropy [14].

There have been works attempting to simplify or approximate CN-FDTD such as, alternating direction implicit (ADI-FDTD) method [15], CN Douglas-Gunn method [16], CN cycle-sweep method [17] and CN approximate factorization splitting method [18]. These approximations suffer up to some extent of numerical errors, which may become severe for some practical applications [19-21]. Recently, both iterative preconditioned/non-preconditioned [22, 23] and direct solving of CN-FDTD [24] have been developed.

Selection of the matrix solver to handle the implicit system of equations arising in CN-FDTD is the critical part to fully harness the advantages of using larger time-step, since the computational costs associated with the solver must be kept as low as possible. Either direct or iterative solvers can be employed. Direct solvers are not the preferred choice for large problems of practical importance because of their enormous memory requirements [24]. Iterative solvers based on Krylov subspace theory have proven to be suitable for practical 
problems $[22,23]$. However the computational efficiency of an iterative solver is application-dependent and therefore determination of the effectiveness of each method is essential.

In this paper, we present a new method to solve the $3 \mathrm{D} \mathrm{CN}$ FDTD method for frequency-dependent media (FD-CN-FDTD), based on Krylov-subspace solvers for sparse systems. One of the future objectives of this work is to observe the interaction of electromagnetic waves with human body. As human tissues have frequency dispersive characteristics [2], frequency dependent media have to be introduced into CN-FDTD. Two most appropriate mathematical models for biological tissues are the Cole-Cole model and the Debye model. Out of these, the Debye model is chosen because it is widely used and easy to implement. Single-pole Debye media have been introduced into CN-FDTD by means of an auxiliary differential formulation [25]. The scheme results in a sparse system of linear equations involving the three components of the electric field, from which all the remaining field quantities are explicitly found. The remaining of this paper is organized as follows: Section 2 introduces FD-CN-FDTD mathematically. The techniques to solve the system and numerical results are presented in Section 3. Section 4 presents the conclusion and discuss on open issues for future work.

\section{THEORY OF FD-CN-FDTD}

Maxwell's curl equations in material independent form are

$$
\begin{aligned}
& \nabla \times \mathbf{E}=-\frac{\partial \mathbf{B}}{\partial t} \\
& \nabla \times \mathbf{H}=\frac{\partial \mathbf{D}}{\partial t}
\end{aligned}
$$

where $\mathbf{E}, \mathbf{H}, \mathbf{D}$ and $\mathbf{B}$ are the electric field, magnetic field, electric flux density and magnetic flux density, respectively. The constitutive relationships for isotropic, linear, non-magnetic, single-pole Debye electrically-dispersive media are, in frequency domain,

$$
\begin{aligned}
& \mathbf{B}=\mu_{0} \mathbf{H} \\
& \mathbf{D}=\epsilon_{0}\left(\epsilon_{\infty}+\frac{\epsilon_{\mathrm{S}}-\epsilon_{\infty}}{1+\jmath \omega \tau_{\mathrm{D}}}-\jmath \frac{\sigma}{\omega \epsilon_{0}}\right) \mathbf{E}
\end{aligned}
$$

where $\epsilon_{0}$ and $\mu_{0}$ are the free-space permittivity and permeability, $\epsilon_{\mathrm{S}}$ is the static permittivity, $\epsilon_{\infty}$ is the optical permittivity, $\tau_{\mathrm{D}}$ is the relaxation time and $\sigma$ is the static conductivity. Eq. (4) can be rewritten as

$$
(\jmath \omega)^{2} \tau_{\mathrm{D}} \mathbf{D}+\jmath \omega \mathbf{D}=(\jmath \omega)^{2} \epsilon_{0} \epsilon_{\infty} \tau_{\mathrm{D}} \mathbf{E}+\jmath \omega\left(\epsilon_{0} \epsilon_{\mathrm{S}}+\sigma \tau_{\mathrm{D}}\right) \mathbf{E}+\sigma \mathbf{E}
$$


By mapping $(\jmath \omega)^{m}$, in frequency domain, into $\frac{\partial^{m}}{\partial t^{m}}$, in time domain, Eq. (5) can be written as

$$
\tau_{\mathrm{D}} \frac{\partial^{2} \mathbf{D}}{\partial t^{2}}+\frac{\partial \mathbf{D}}{\partial t}=\epsilon_{0} \epsilon_{\infty} \tau_{\mathrm{D}} \frac{\partial^{2} \mathbf{E}}{\partial t^{2}}+\left(\epsilon_{0} \epsilon_{\mathrm{S}}+\sigma \tau_{\mathrm{D}}\right) \frac{\partial \mathbf{E}}{\partial t}+\sigma \mathbf{E}
$$

Application of the Crank-Nicolson method [24, 26] to Eqs. (1), (2), (3), and (6), after algebraic manipulation, yields an equation only with electric field $E^{n+1}$ terms:

$$
\begin{aligned}
& E_{x}{ }^{n+1}-\frac{\xi_{1}}{\xi_{4}}\left(\frac{\Delta t}{2}\right)^{2} \frac{1}{\mu} \frac{\partial^{2} E_{x}{ }^{n+1}}{\partial y^{2}}+\frac{\xi_{1}}{\xi_{4}}\left(\frac{\Delta t}{2}\right)^{2} \frac{1}{\mu} \frac{\partial^{2} E_{y}{ }^{n+1}}{\partial x \partial y} \\
& +\frac{\xi_{1}}{\xi_{4}}\left(\frac{\Delta t}{2}\right)^{2} \frac{1}{\mu} \frac{\partial^{2} E_{z}{ }^{n+1}}{\partial z \partial x}-\frac{\xi_{1}}{\xi_{4}}\left(\frac{\Delta t}{2}\right)^{2} \frac{1}{\mu} \frac{\partial^{2} E_{x}{ }^{n+1}}{\partial z^{2}} \\
= & \frac{\xi_{1}}{\xi_{4}} D_{x}{ }^{n}+\frac{\xi_{1}}{\xi_{4}} \frac{\Delta t}{2} \frac{\partial H_{z}{ }^{n}}{\partial y}+\frac{\xi_{1}}{\xi_{4}}\left(\frac{\Delta t}{2}\right)^{2} \frac{1}{\mu} \frac{\partial^{2} E_{x}{ }^{n}}{\partial y^{2}} \\
& -\frac{\xi_{1}}{\xi_{4}}\left(\frac{\Delta t}{2}\right)^{2} \frac{1}{\mu} \frac{\partial^{2} E_{y}{ }^{n}}{\partial x \partial y}-\frac{\xi_{1}}{\xi_{4}} \frac{\Delta t}{2} \frac{\partial H_{y}{ }^{n}}{\partial z}-\frac{\xi_{1}}{\xi_{4}}\left(\frac{\Delta t}{2}\right)^{2} \frac{1}{\mu} \frac{\partial^{2} E_{z}{ }^{n}}{\partial z \partial x} \\
& +\frac{\xi_{1}}{\xi_{4}}\left(\frac{\Delta t}{2}\right)^{2} \frac{1}{\mu} \frac{\partial^{2} E_{x}{ }^{n}}{\partial z^{2}}+\frac{\xi_{1}}{\xi_{4}}\left(\frac{\Delta t}{2}\right)^{2} \frac{\partial H_{z}{ }^{n}}{\partial y}-\frac{\xi_{1}}{\xi_{4}}\left(\frac{\Delta t}{2}\right)^{2} \frac{\partial H_{y}{ }^{n}}{\partial z} \\
& +\frac{\xi_{2}}{\xi_{4}} D_{x}{ }^{n}+\frac{\xi_{3}}{\xi_{4}} D_{x}{ }^{n-1}-\frac{\xi_{5}}{\xi_{4}} E_{x}{ }^{n}-\frac{\xi_{6}}{\xi_{4}} E_{x}{ }^{n-1}
\end{aligned}
$$

where $\xi_{1}, \xi_{2}, \xi_{3}, \xi_{4}, \xi_{5}, \xi_{6}$ are space dependent and functions of the Debye parameters and $\Delta t[24]$.

Cyclic permutation of $x, y$ and $z$ in Eq. (7) yields the remaining two $E$-field equations. By applying them to each Yee-grid position, a system of linear equations of $\mathbf{A N}=\mathbf{C}$ is set up. $\mathbf{A}$ is the coefficient matrix, $\mathbf{N}$ represents a vector with the electric field components to be solved, and $\mathbf{C}$ is the excitation vector. The system of equations $\mathbf{A N}=\mathbf{C}$ is solved first to find the $\mathbf{E}$ field. $\mathbf{D}$ and $\mathbf{H}$ fields are calculated in an explicit manner from the $\mathbf{E}$ field. In order to terminate the computational space Mur first order boundary conditions are employed in this work.

\section{SOLUTION OF FD-CN-FDTD}

\subsection{Sparse Matrix}

The coefficient matrix A generated by FD-CN-FDTD is highly sparse. The size of $\mathbf{A}$ is $9\left(N_{x}\right)^{2}\left(N_{y}\right)^{2}\left(N_{z}\right)^{2}$ where $N_{x}, N_{y}, N_{z}$ are the size of the computational space in $x, y$ and $z$ directions. When the 
problem space is homogeneous, the coefficient matrix is symmetric and otherwise asymmetric. Fig. 1 shows the sparsity pattern of $\mathbf{A}$ in the case where the entire computational space is filled with Debye parameters $\epsilon_{\mathrm{S}}=6.2, \epsilon_{\infty}=3.5, \sigma=0.029 \mathrm{~S} / \mathrm{m}$ and $\tau_{\mathrm{D}}=39.0 \mathrm{ps}$. The sparsity pattern of Fig. 1 is similar to that of three dimensional Finite Difference Frequency Domain (FDFD) method [27], and therefore the discussions in this paper could also be useful to the FDFD researchers.

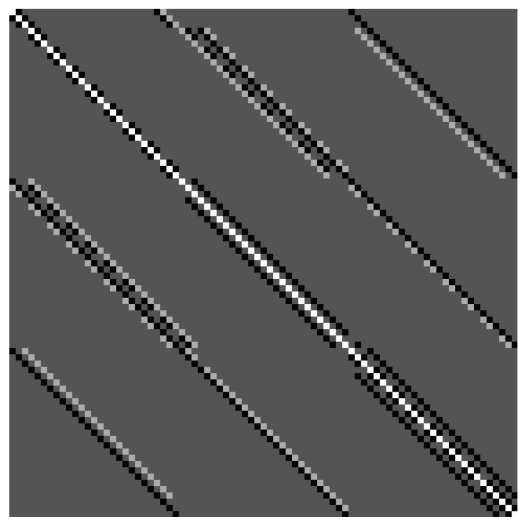

Figure 1. Sparsity pattern of coefficient matrix A of FD-CN-FDTD. The pixel in white has highest value. One in light gray has the value of $1 / 16$ of the one in white. One in dark gray has the value of zero. One in black has the negative value of the value in light gray.

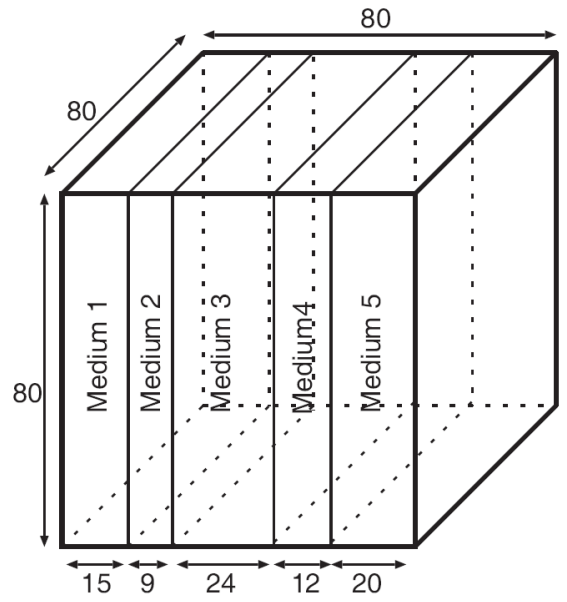

\begin{tabular}{|c|c|c|c|c|}
\hline Medium & $\epsilon_{\mathrm{s}}$ & $\epsilon_{\infty}$ & $\tau(\mathrm{ps})$ & $\sigma(\mathrm{S} / \mathrm{m})$ \\
\hline Medium1 & 71.66 & 34.58 & 5.65 & 0.021 \\
\hline Medium2 & 6.2 & 3.5 & 39.0 & 0.029 \\
\hline Medium3 & 9.5 & 4.2 & 77.0 & 0.037 \\
\hline Medium4 & 87.34 & 49.13 & 26.89 & 0.045 \\
\hline Medium5 & 4.8 & 2.8 & 7.0 & 0.053 \\
\hline
\end{tabular}

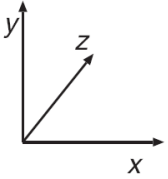

Figure 2. Computational environment for numerical studies using FD-CN-FDTD. 
In this paper, two cases are considered for study. The first one consists of an inhomogeneous medium, in a cubic space of size $80^{3}$ cells, with 5 different media as shown in Fig. 2. The second one involves the same cubic space of the previous case, now filled with a homogeneous medium with Debye parameters $\epsilon_{\mathrm{S}}=6.2, \epsilon_{\infty}=3.5, \sigma=0.029 \mathrm{~S} / \mathrm{m}$ and $\tau_{\mathrm{D}}=39.0 \mathrm{ps}$. In both cases a $z$-directed dipole hard source with a time variation given by a Gaussian pulse centered at $6.9 \mathrm{GHz}$, with $4.94 \mathrm{GHz}$ bandwidth ( $-3 \mathrm{~dB}$ decay) was placed at the centre of the computational space. Spatial sampling was uniform: $\Delta x=\Delta y=$ $\Delta z=\Delta=10^{-3} \mathrm{~m}$. The time step is taken equal or above the CFL stability condition of the explicit FDTD: $\Delta t=C F L N \times \Delta /(c \sqrt{3})$ with $C F L N$ henceforth referred to as CFL number, and $c$ the freespace light-speed. The level of accuracy in waveform compared with explicit frequency-dependent FDTD is the same as the one presented in Fig. 2 in [24].

\subsection{Condition Number and Diagonal Dominance}

The ease of solution of a linear system of equations can be measured by the condition number of $\mathbf{A}$. [28] reports that the condition number of the coefficient matrix $\mathbf{A}$ for frequency-independent Crank-Nicolson scheme increases with the CFL number, which naturally also applies to our FD-CN-FDTD method. Hence for high $C F L N$, the matrix becomes severely ill-conditioned, requiring high computation time to be solved by iterative methods.

Similar conclusions are found by realizing that the diagonal dominance of the coefficient matrix ${ }^{\dagger}$ improves when $C F L N$ decreases, leading to matrices which are easier to solve. Fig. 3 shows how the absolute values of diagonal and the sum of absolute values of offdiagonal elements of $\mathbf{A}$ vary with $C F L N$, both for the homogeneous and inhomogeneous cases previously described. A representative row of $\mathbf{A}$ has been taken, corresponding to the interior computational space, which comprises nearly the whole coefficient matrix (except the boundary-contributed rows). For low CFLN all the rows of the coefficient matrix are strictly diagonally dominant except a very few which are contributed by the boundary cases, whereas high $C F L N$ deteriorates this property. Fig. 3 shows that the advantageous diagonal property of the coefficient matrix is lost with increased $C F L N$ irrespective of the media parameters or homogeneity, The diagonaldominance criterion is simpler to handle than the condition-number one, which is intensive to compute in practice. An additional advantage

\footnotetext{
$\dagger$ A matrix is said to be diagonally dominant if $\left|a_{i, i}\right| \geq \sum_{\substack{j=1 \\ j \neq i}}^{n}\left|a_{i, j}\right|$ for all the $i$ rows.
} 


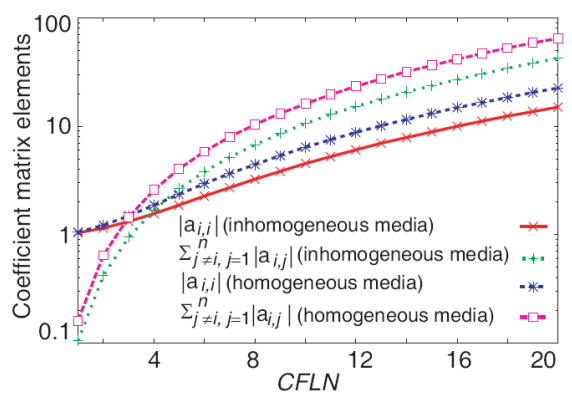

Figure 3. Absolute values of diagonal and sum of absolute values of off-diagonal entries of the coefficient matrix changes with $C F L N$.

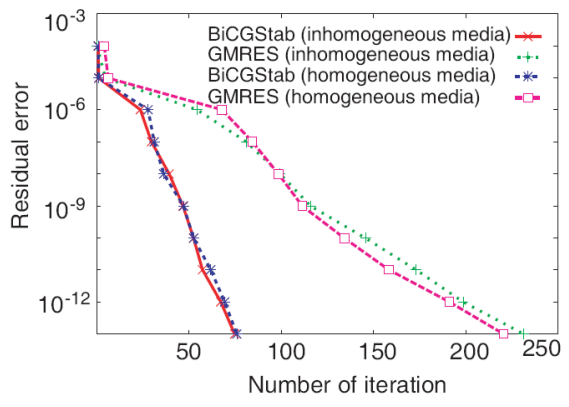

Figure 4. Residual error versus number of average iteration required by BiCGStab and GMRES for homogeneous and inhomogeneous cases when $C F L N=20$.

of the diagonal dominance criterion is that it points us a direction to research in the building of appropriate preconditioners to alleviate this problem.

\subsection{Iterative Solvers}

To solve FD-CN-FDTD either direct or iterative solvers can be used. Although direct solvers are robust and reliable [24], they are computationally more expensive than iterative solvers, unless parallelized, and require excessively large memory. For example, a $30^{3}$ cells computational space solved by the direct solver in double precision using sparse Gaussian elimination requires $2.4 \mathrm{~GB}$ of memory whereas iterative solvers like Bi-Conjugate Gradient Stabilised (BiCGStab) and Generalised Minimal Residual (GMRES) require only $62 \mathrm{MB}$ and $65 \mathrm{MB}$, respectively. Thus for practical problems iterative solvers should be used [29].

Depending on the homogeneity of the computational space FDCN-FDTD yields symmetric and asymmetric matrices. Since for homogeneous problems the coefficient matrix is symmetric, iterative solvers like Conjugate Gradient (CG) method can be used. However practical problems are inhomogeneous and we have to resort to iterative solvers for asymmetric matrices. Usual ones are GMRES, BiCGStab or Conjugate Gradient Squared (CGS) (Appendix D of [30]). CGS suffers from irregular convergence, which may lead to substantial build-up of rounding errors, because it is based on squaring the residual polynomial $[29,30]$. Therefore, this study focuses only in GMRES and BiCGStab. An advantage of BiCGStab method is that 
its computational cost per iteration is similar to that of CGS but it avoids the irregular convergence patterns of CGS while maintaining about the same rate of convergence. GMRES is said to be a very robust solver for nonsymmetric matrices. It leads to the smallest residual for a fixed number of iteration steps. But these steps become increasingly expensive and in order to limit the increasing storage requirements and work per iteration step, restarting is necessary. In this work GMRES(m) that restarts every $m$ iterations [31] and BiCGStab of [32] were used.

\subsection{Performance Study of BiCGStab and GMRES}

In this section we compare the performance of BiCGStab and GMRES both for the homogeneous and inhomogeneous media cases described earlier. Fig. 4 shows the convergence pattern for $C F L N=20$, plotting the residual error as a function of the average number of iterations required to achieve a specified accuracy. For example, to make the residual error lower than $10^{-8}$ BiCGStab requires about 45 iterations whereas GMRES requires about 97 iterations in both homogeneous and inhomogeneous cases. The convergence rate of the solvers is weakly affected by homogeneity. For a modest value of $C F L N$ iteration numbers would certainly be lowered than those showed in Fig. 4 $(C F L N=20)$.

Figure 5 shows how the average number of iterations, required by BiCGStab and GMRES to converge, increases with the CFL number. Stopping criteria in this case was $10^{-13}$ and the reason for selecting this small value of convergence tolerance is, in FD-CN-FDTD, unlike

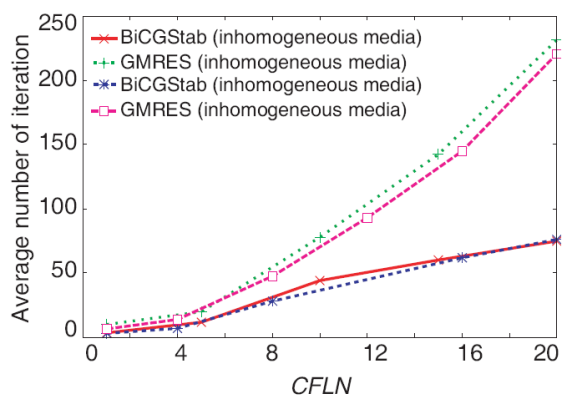

Figure 5. Average number of iterations required for different CFL numbers with residual error of $10^{-13}$.

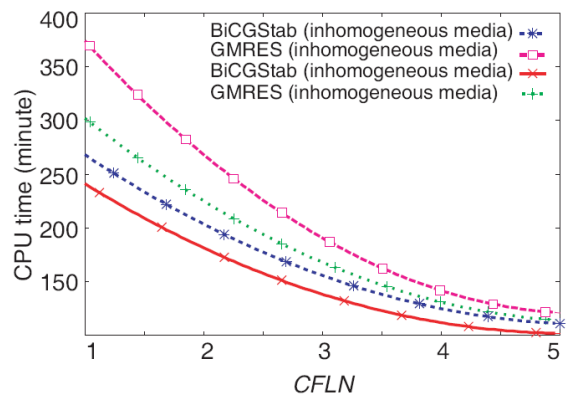

Figure 6. CPU time required by BiCGStab and GMRES for different $C F L N$. 
frequency-independent CN-FDTD, $\mathbf{D}=\epsilon \mathbf{E}$ is used and therefore $\mathbf{D}$ can have a value of such small order because of $\epsilon$ (permitivitty). GMRES stagnates when convergence tolerance is below $10^{-13}$ while BiCGStab can work even at a lower convergence tolerance. Both solvers require more iterations to converge as $C F L N$ goes up but the rate of increase of iteration numbers with $C F L N$ is higher for GMRES than for BiCGStab. Homogeneity does not affect significantly this rate, particularly, for BiCGStab. The change of iteration number with $C F L N$ for convergence tolerance values from $10^{-12}$ to $10^{-3}$ can be assumed from Fig. 4. In FD-CN-FDTD, the total number of iterations required to complete the simulation decreases with $C F L N$, but the increase of computational costs per iteration with $C F L N$, as shown in Fig. 5, can undermine this positive effect unless the solution is very efficient.

Figure 6 plots the CPU time required by BiCGStab and GMRES as a function of $C F L N$. The case of stopping criterion of $10^{-13}$ was employed and simulated to reach a fixed time instant by letting the code run for $1200 / C F L N$ time steps on a dual AMD Opteron 280 with $8 \mathrm{~GB}$ of memory. Observe that the CPU-time decreases with $C F L N$, for both solvers, although GMRES requires more CPU time than BiCGStab.

Table 1 presents the memory required by the two solvers for three different computational space sizes. GMRES always requires more memory than BiCGStab.

From all the above, we can conclude that BiCGStab outperforms GMRES in computational efficiency. This finding is in contrary to that of [28] which reports GMRES is the fastest for the frequencyindependent CN-FDTD scheme presented there. The work of [28] is based on Eqs. (1) and (2) while FD-CN-FDTD additionally involves Eq. (6) which has 2nd order time derivative terms. The FD-CN-FDTD involves nine field components in place of six for CNFDTD and the sparsity pattern of the former has more bands than the latter [23]. Apart from this, the concerned problem of simulation, implementation, optimization and parameters tuning have an obvious influence in concluding which solver is the most efficient.

Table 1. Memory required by BiCGStab and GMRES for different computational spaces.

\begin{tabular}{|c|c|c|c|}
\hline Computational Size (cells) & $40^{3}$ & $60^{3}$ & $80^{3}$ \\
\hline BiCGStab & $145 \mathrm{MB}$ & $487 \mathrm{MB}$ & $1.1 \mathrm{~GB}$ \\
\hline GMRES & $151 \mathrm{MB}$ & $507 \mathrm{MB}$ & $1.2 \mathrm{~GB}$ \\
\hline
\end{tabular}


Finally, we have also implemented two preconditioners to solve for FD-CN-FDTD: Incomplete LU with no fill-in or ILU(0) and Sparse Approximate Inverse (SAI). ILU(0) did not give any improvement in convergence (rather convergence deteriorated). However SAI gave slight improvement in reducing the number of iterations to converge but there were two major setbacks making it unsuitable for use. The time to compute the approximate inverse preconditioner is too large which makes the total CPU time longer than that without any proconditioner. [23] showed SAI can reduce iteration numbers but did not mention the total CPU time. A second problem is that memory requirements of SAI restricted the maximum computational space to only $30^{3}$ cells. SAI also showed lack of robustness in our case.

\section{CONCLUSION}

In this paper, we have presented a new Krylov-based approach to solve the three dimensional CN-FDTD method for frequency-dependent Debye dispersive media. Two best-known iterative methods, GMRES and BiCGStab, were compared in terms of the number of iteration requirements for convergence with different CFLN, CPU-time and memory requirements. BiCGStab outperforms GMRES when used with FD-CN-FDTD in every aspect of the study. It has also been pointed out that the degradation of diagonal-dominance of the coefficient matrix with increased $C F L N$ is a main reason for the increase of the CPU time needed by the solvers. Furthermore it was found that ILU(0) and SAI preconditioners can not improve the computational efficiency of FD-CN-FDTD. Many of these findings about frequency-dependent CN-FDTD do not match with the existing literature on frequency-independent CN-FDTD and possible reasons for this are also mentioned. Further work is needed to tailor suitable preconditioners to improve the iterative solver convergence.

\section{ACKNOWLEDGMENT}

This work has been partially supported by the EU FP7 under GA 205294, and by the Spanish National Projects TEC2007-66698-C04-02, CSD2008-00068, DEX-530000-2008-105, and the Junta de Andalucia Project TIC1541.

\section{REFERENCES}

1. Yee, K. S., "Numerical solution of initial boundary value problems involving maxwell's equations in isotropic media," IEEE Trans. 
Antennas Propagat., Vol. 14, No. 3, 302-307, 1966.

2. Taflove, A. and S. Hagness, Computational Electromagnetics. The Finite-difference Time-domain Method, Artech House, Boston, MA, 2005.

3. Sullivan, D. M., "Frequency-dependent FDTD methods using Ztransforms," IEEE Trans. Antennas Propagat., Vol. 40, 12231230, 1992.

4. Luebbers, R. J., F. Hunsberger, K. S. Kunz, R. B. Standler, and M. Schneider, "A frequency-dependent finite-difference timedomain formulation for dispersive materials," IEEE Trans. Electromagn. Compat., Vol. 32, No. 3, 220-227, 1990.

5. Zhang, Y.-Q. and D.-B. Ge, "A unified fdtd approach for electromagnetic analysis of dispersive objects," Progress In Electromagnetics Research, PIER 96, 155-172, 2009.

6. Prokopidis, K. P., E. P. Kosmidou, and T. D. Tsiboukis, "An FDTD algorithm for wave propagation in dispersive media using higher-order schemes," Journal of Electromagnetic Waves and Applications, Vol. 18, No. 9, 1171-1194, 2004.

7. Taflove, A. and S. Hagness, Computational Electrodynamics: The Finite-difference Time-domain Method, 3rd edition, Artech House, Boston, MA, 2005.

8. Cangellaris, A. C. and R. Lee, "On the accuracy of numerical wave simulations based on finite methods," Journal of Electromagnetic Waves and Applications, Vol. 6, No. 12, 1635-1653, 1992.

9. Castillo, S. and S. Omick, "Suppression of dispersion in FDTD solutions of Maxwell's equations," Journal of Electromagnetic Waves and Applications, Vol. 8, No. 9-10, 1193-1221, 1994.

10. Garcia, S. G., F. Costen, M. F. Pantojal, A. Brown, and A. R. Bretones, "Open issues in unconditionally stable schemes," Progress In Electromagnetics Research Symposium Abstracts, 2009.

11. Kung, F. and H. T. Chuah, "Stability of classical finite-difference time-domain (FDTD) formulation with nonlinear elements A new perspective," Journal of Electromagnetic Waves and Applications, Vol. 17, No. 9, 1313-1314, 2003.

12. Liang, F. and G. Wang, "Fourth-order locally one-dimensional FDTD method," Journal of Electromagnetic Waves and Applications, Vol. 22, No. 14-15, 203-2043, 2008.

13. Crank, J. and P. Nicolson, "A practical method for numerical evaluation of solutions of partial differential equations of the heatconduction type," Proc. Camb. Phil. Soc., Vol. 43, 50-67, 1947. 
14. Tan, E. L., "Efficient algorithms for Crank Nicolson based finite difference time domain methods," IEEE Trans. Microwave Theory and Tech., Vol. 56, No. 2, 408-413, February 2008.

15. Namiki, T., "A new FDTD algorithm based on alternatingdirection implicit method," IEEE Trans. Microwave Theory and Tech., Vol. 47, 2003-2007, 1999.

16. Sun, G. and C. Trueman, "Unconditionally stable Crank-Nicolson scheme for solving two dimensional Maxwell's equations," Electronics Lett., Vol. 39, No. 7, 595-597, 2003.

17. Sun, G. and C. Trueman, "Efficient implementations of the CrankNicolson scheme for the finite-difference time-domain method," IEEE Trans. Microwave Theory and Tech., Vol. 54, No. 5, 22752284, May 2006.

18. Sun, G. and C. Trueman, "Unconditionally-stable FDTD method based on Crank-Nicolson scheme for solving three dimensional Maxwell's equations," Electronics Lett., Vol. 40, No. 10, 589-590, 2004.

19. Garcia, S. G., T. Lee, and S. C. Hagness, "On the accuracy of the ADI-FDTD method," Antennas and Wireless Propagat. Lett., Vol. 1, No. 1, 31-34, 2002.

20. Garcia, S. G., R. G. Rubio, A. R. Bretones, and R. G. Martin, "On the dispersion relation of ADI-FDTD," IEEE Microwave and Wireless Comp. Lett., Vol. 16, 354-356, June 2006.

21. Garcia, S. G., F. Costen, A. R. Bretones, and A. Brown, "Stateof-the-art in unconditionally stable FDTD schemes," IEEEAP/URSI 2007, July 2007.

22. Yang, Y., R. Chen, and E. Yung, "The unconditionally stable Crank Nicolson FDTD method for three-dimensional Maxwell's equations," Microwave and Opt. Tech. Lett., Vol. 48, 1619-1622, 2006.

23. Yang, Y., Z. Fan, D. Ding, and S. Liu, "Application of the preconditioned GMRES to the Crank-Nicolson finite-difference time-domain algorithm for 3D full-wave analysis of planar circuits," Microwave and Opt. Tech. Lett., Vol. 50, 1458-1463, 2008.

24. Rouf, H. K., F. Costen, and S. G. Garcia, "3-D Crank-Nicolson finite difference time domain method for dispersive media," Electronics Lett., Vol. 45, No. 19, 961-962, September 10, 2009.

25. Joseph, R., S. Hagness, and A. Taflove, "Direct time integration of Maxwell's equations in linear dispersive media with absorption for scattering and propagation of femtosecond electromagnetic 
pulses," Optics Lett., Vol. 16, No. 18, 1412-1414, 1991.

26. Rubio, R. G., S. G. Garcia, A. R. Bretones, and R. G. Martin, "Crank-nicolson reformulation of ADI-FDTD PML extensions," Antennas and Wireless Propagat. Lett., Vol. 5, No. 1, 357-360, 2006.

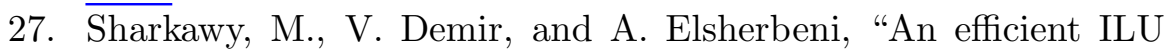
preconditioning for highly sparse matrices constructed using the FDFD method," IEEE Antennas and Propagat. Magazine, Vol. 49, No. 6, 135-139, 2007.

28. Yang, Y., R. Chen, D. Ding, Z. Fan, and E. Yung, "Application of iterative solvers in 3D Crank-Nicolson FDTD method for simulating resonant frequencies of the dielectric cavity," AsiaPacific Microwave Conference, 1-4, 2007.

29. Saad, Y., Iterative Methods for Linear Systems, 297, PWS Publishing, 1996.

30. Barrett, R., M. W. Berry, T. F. Chan, J. Demmel, J. Donato, J. Dongarra, V. Eijkhout, R. Pozo, C. Romine, and H. Van Der Vorst, Templates for the Solution of Linear Systems: Building Blocks for Iterative Methods, 117, SIAM Press, Philadelphia, 1993.

31. Saad, Y. and M. H. Schultz, "GMRES: A generalized minimal residual method for solving nonsymmetric linear systems," SIAM J. Sci. Stat. Comput., Vol. 7, 856-869, 1986.

32. Van Der Vorst, H., "Bi-CGSTAB: A fast and smoothly converging variant of Bi-CG for the solution of nonsymmetric linear systems," SIAM J. Sci. Stat. Comput., Vol. 13, 631-644, 1992. 